Internat. J. Math. \& Math. Sci.

Vol. 2 No. 2 (1979) 223-228

\title{
A GRAPH AND ITS COMPLEMENT WITH SPECIFIED PROPERTIES I: CONNECTIVITY
}

\author{
JIN AKIYAMA * \\ Mathematics Department \\ Nippon Ika University \\ Kawasaki, Japan \\ FRANK HARARY * * \\ Mathematics Department \\ University of Michigan \\ Ann Arbor, Michigan 48109 \\ (Received November 14, 1978) \\ Dedicated to Kar1 Menger
}

ABSTRACT. We investigate the conditions under which both a graph $G$ and its complement $\bar{G}$ possess a specified property. In particular, we characterize all graphs $G$ for which $G$ and $\bar{G}$ both (a) have connectivity one, (b) have line-connectivity one, (c) are 2-connected, (d) are forests, (e) are bipartite, (f) are outerplanar and (g) are eulerian. The proofs are elementary but amusing. KEY WORDS AND PHRASES. Graphs, Complement. AMS (MOS) SUBJECT CLASSIFICATION (1970) CODES. $05 C 99$.

*Visiting Scholar 1978-79 at The University of Michigan.

** Vice-President, Calcutta Mathematical Society, 1978 and 1979. 


\section{CONNECTIVITY.}

The connectivity (or line-connectivity) $\kappa=\kappa(G)$ (or $\lambda=\lambda(G)$ ) of a graph $G$ is the minimum number of points (or lines) whose removal results in a disconnected or a trivial graph. We write $\bar{K}$ (or $\bar{\lambda}$ ) for $K(\bar{G})$ (or $\lambda(\bar{G})$ ) where $\bar{G}$ is the complement of $\mathrm{G}$. We follow the graph theoretic terminology and notation of the book [1]. Recall that $\Delta$ denotes the maximum degree among all points of $\mathrm{G}$.

LEMMA 1. The complement $\bar{G}$ of a connected graph $G$ is connected if and only if $G$ has no spanning complete bipartite subgraph.

PROOF. If $G$ has a spanning complete bipartite subgraph, then $\bar{G}$ clearly contains no line joining the two parts, hence must be disconnected. Conversely, if $\bar{G}$ is disconnected, then any bipartition of $V(G)$ in which one part consists of the points of precisely one component of $\bar{G}$ gives a spanning complete bipartite subgraph of $G$.

The next statement is an easy consequence of the lemma.

THEOREM 1. A graph $G$ with $p$ points satisfies the condition $K=\bar{K}=1$ if and only if $G$ is a graph with either

(1) $K=1$ and $\Delta=p-2$, or

(2) $K=1, \Delta \leq p-3$ and $G$ has a cutpoint $v$ with endline $e$ and endpoint $u$ such that G - u contains a spanning complete bipartite subgraph.

PROOF. We note that if $K=\bar{K}=1$, then the degree of each point of $G$ is at most $\mathrm{p}-2$, since otherwise $\bar{G}$ would contain an isolated point which would make $\bar{K}=0$.

(1) Let $G$ be a graph with $\Delta=p-2$ and $K=1$, as in Figure 1a.

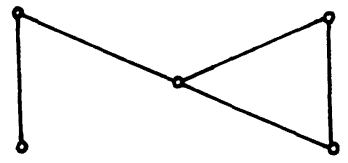

(a)
Figure 1.

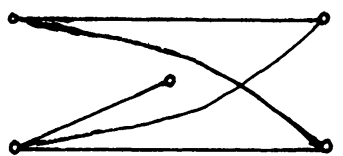

(b) 
The removal of any cutpoint $v$ from $G$ results in a disconnected graph, so that $\overline{\mathrm{G}-\mathrm{V}}$ is connected. Since $\Delta=\mathrm{p}-2$ by hypothesis, $\mathrm{v}$ is adjacent in $\overline{\mathrm{G}}$ to a point of $\overline{G-v}$. Thus $\bar{G}$ is connected. Furthermore $\bar{G}$ has an endline since $\Delta=$ $\mathrm{p}-2$, and hence $\overline{\mathrm{G}}$ has a cutpoint (as illustrated in Figure $1 \mathrm{~b}$ ), so that $\bar{\kappa}=1$. (2) Let $G$ be a graph with $k=\bar{k}=1$ and $\Delta \leq p-3$. By the definition of $k$, $G$ is connected and has a cutpoint $v$. We see that $H=G-v$ has just two components, since otherwise every two points of $\bar{G}$ would lie on a common cycle of $\bar{G}$ and thus $\bar{G}$ would have no cutpoint, contradicting $\bar{K}=1$. Denote by $\mathrm{H}_{1}$ and $\mathrm{H}_{2}$ the two components of $\mathrm{H}$, with $\mathrm{p}_{1}$ and $\mathrm{p}_{2}$ points respectively. Assume that both $p_{1}, p_{2} \geq 2$. Then $\bar{G}$ would have no cutpoint since every two points of $\bar{G}$ would lie on a common cycle of $\bar{G}$. Thus it is sufficient to consider only a connected graph $\mathrm{G}$ which has a cutpoint with endline e and endpoint $u$. We now show that $G-u$ contains a spanning complete bipartite subgraph. If $G-u$ does not contain such a subgraph, then $\overline{G-U}$ is connected by Lemma 1. Moreover, the endpoint $u$ of $e$ is adjacent in $\bar{G}$ to every point of $\bar{G}$ lie on a common cycle and so $\bar{G}$ has no cutpoint, which again contradicts $\bar{K}=1$. Thus $G-u$ contains a spanning complete bipartite subgraph.

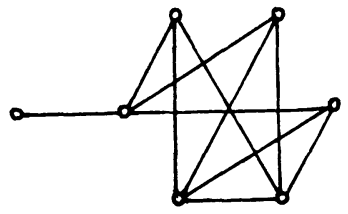

(a)
Figure 2.

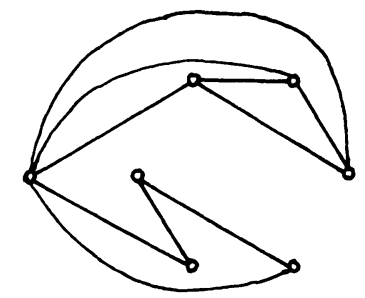

(b)

Conversely, let $G$ satisfy the condition (2) as shown in Figure $2 a$. Then $\bar{G}$ is connected and the removal of the endpoint $u$ from $\bar{G}$ results in at least two components by Lemma 1. Hence we see that $k=\bar{K}=1$.

A graph $G$ is a block if $G$ is connected and has no cutpoint. From Theorem 1 and Lemma 1, we obtain two consequences whose proofs are ommitted or outlined. 
COROLLARY 1a. If $G$ is a block, then $\bar{G}$ is also a block if and only if

(1) $2 \leq \operatorname{deg} v \leq \mathrm{p}-3$ for every point $\mathrm{v}$ of $\mathrm{G}$, and

(2) G has no spanning complete bipartite subgraph.

CORLLARY 1b. A graph $G$ with $p$ points satisfies the condition $\lambda=\lambda$

$=1$ if and only if $G$ is a connected graph with a bridge and $\Delta=p-2$.

PROOF. Let $G$ be a graph with $\lambda=\bar{\lambda}=1$. Then $G$ satisfies the condition $K=\bar{K}=1$ by the relation $K \leq \lambda$. Hence the graph $G$ satisfies either (1) or (2) of Theorem 1. It is clear that (2) cannot hold, since $\bar{G}$ can possess an endline only if the spanning bipartite subgraph of $\mathrm{G}-\mathrm{u}$ is a star, in which case $\Delta=p-2$, and so (1) must obtain.

Conversely, if $G$ is a graph with $\lambda=1$ and $\Delta=p-2$, then $\bar{G}$ is connected and has an endline, that is, $\bar{\lambda}=1$.

\section{BIPARTITE GRAPHS AND OUTERPLANAR GRAPHS.}

A graph $G$ is a forest if $G$ has no cycles. An outerplanar graph is planar and can be embedded in the plane so that all its points lie on the same face.

THOEREM 2. All the graphs $G$ such that both $G$ and $\bar{G}$ are bipartite are: are shown in Figure 3.

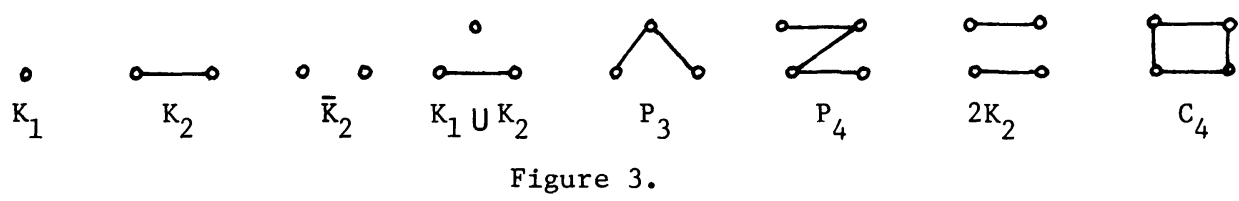

PROOF. The number $k$ of components of $G$ is at most two, since otherwise $\bar{G}$ would contain a triangle.

$\operatorname{CASE} 1: k=2$. Let $G$ have components $G_{1}$ and $G_{2} \cdot$ Both $G_{1}$ and $G_{2}$ are complete, since otherwise $\bar{G}$ would contain a triangle. Furthermore, the order of each of the complete graphs $G_{1}$ and $G_{2}$ is at most two, since otherwise $G$ would contain a triangle. Hence we obtain $G=\bar{K}_{2}, K_{1} U K_{2}$ and $2 K_{2}$. 
CASE 2: $k=1$. Since $G$ is bipartite, the point set of $G$ can be partitioned into two subsets $v_{1}$ and $v_{2}$ such that every line of $G$ joins $v_{1}$ with $v_{2}$. The cardinalities of $v_{1}$ and $v_{2}$ are at most two, since otherwise $\bar{G}$ would contain a triangle. Furthermore, each subgraph induced by any three points of $G$ contains one or two lines. Hence we get $G=K_{1}, K_{2}, P_{3}, P_{4}$, and $C_{4}$.

COROLLARY 2a. All the graphs $G$ such that both $G$ and $\bar{G}$ are forests are:

$$
G=K_{1}, K_{2}, \bar{K}_{2}, K_{1} \cup K_{2}, P_{3} \text { and } P_{4}
$$

We have determined in Theorem 2 all eight graphs such that both $G$ and $\bar{G}$ are bipartite, and note that for none of these graphs $G$ is both $G$ and $\bar{G}$ have even cycles. We now show that for just two graphs $G$, both $G$ and $\bar{G}$ have an odd cycle.

THEOREM 3. The two self-complementary graphs of order $5, A$ and $C_{5}$, are the only $G$ such that both $G$ and $\bar{G}$ have only odd cycles (Figure 4 ).

PROOF. If the number of points of $G$ is at least 6 , either $G$ or $\bar{G}$ contains $\mathrm{C}_{4}$ since the ramsey number $\mathrm{r}\left(\mathrm{C}_{4}\right)=6$. It is easily verified that the two self-complementary graphs of order $5, A$ and $C_{5}$ shown in Figure 4 , are the only $G$ such that both $G$ and $\bar{G}$ have odd cycles.

THEOREM 4. All the graphs $G$ such that neither $G$ nor $\bar{G}$ are forests but both are outerplanar are the following 32 graphs:

(1) the two self-complementary graphs $A$ and $C_{5}$ of order 5 (Figure 4), and

(2) the 15 graphs shown in Figure 5 and their complements.

THEOREM 5. Both $G$ and $\bar{G}$ are eulerian if and only if both are connected, $P$ is odd, and $G$ is eulerian.

of course $p$ must be odd so that the degree of each point in both $G$ and $\bar{G}$ is even. Lemma 1 already gives a simple condition for both $G$ and $\bar{G}$ to be connected. The result follows at once. 

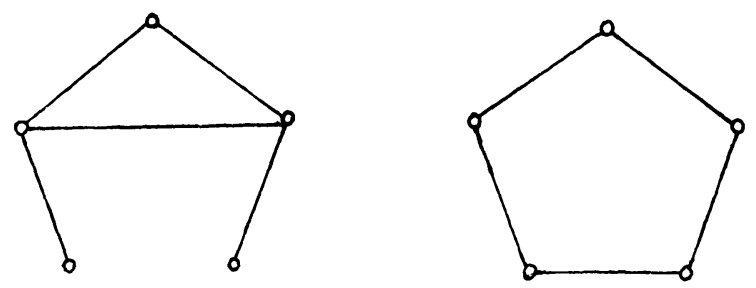

Figure 4.
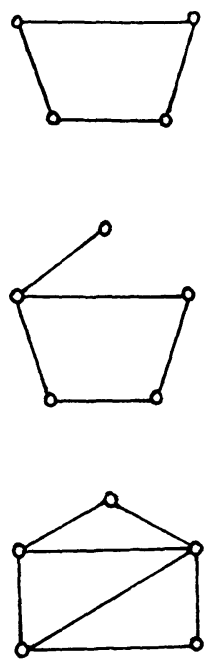

0

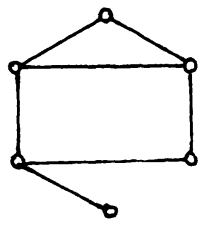

0
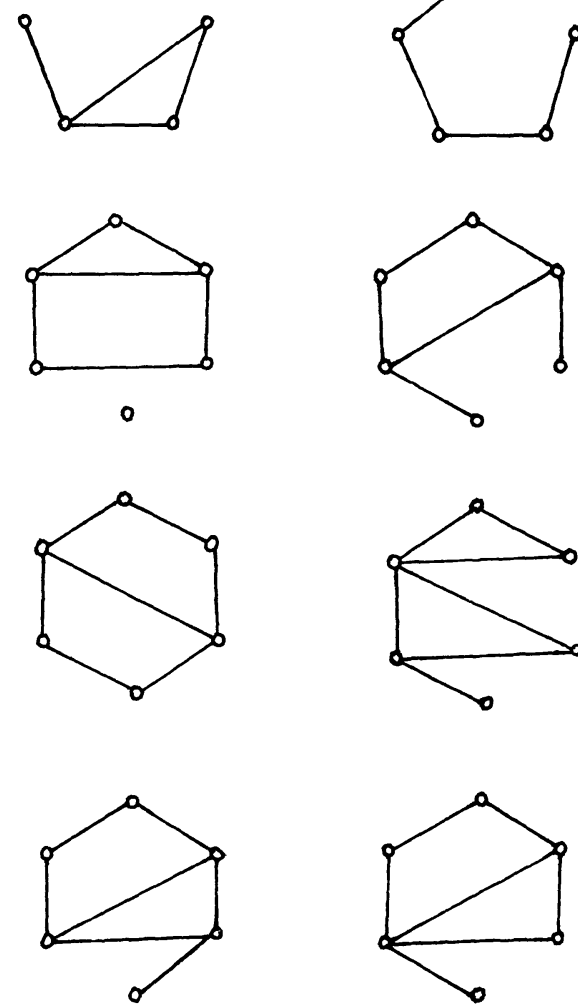
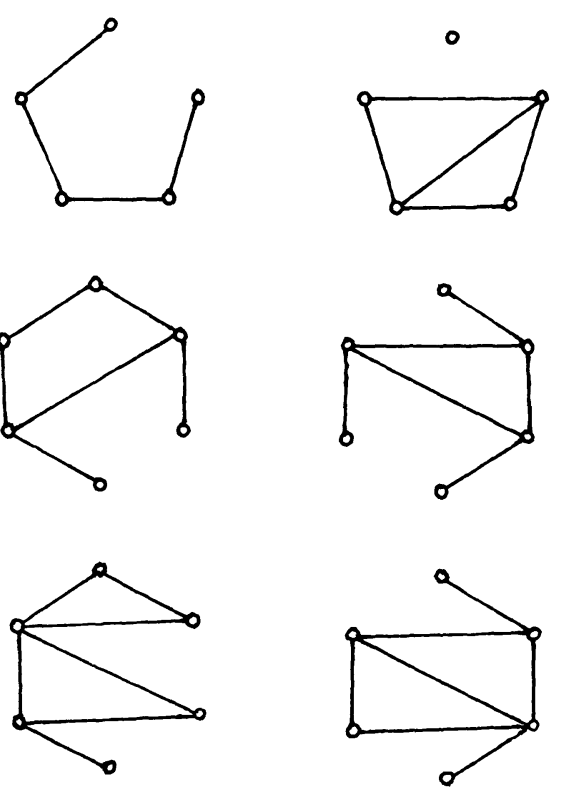

Figure 5.

REFERENCE

1. Harary, F. Graph Theory. Addison-Wesley, Reading, 1969. 


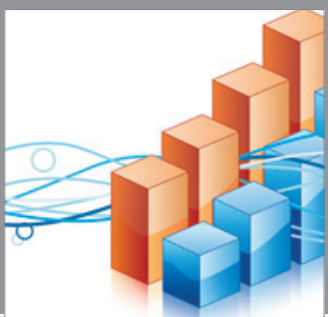

Advances in

Operations Research

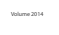

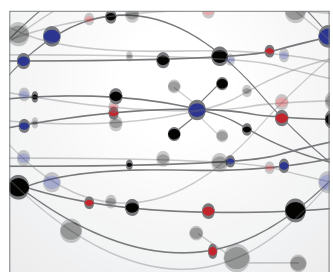

\section{The Scientific} World Journal
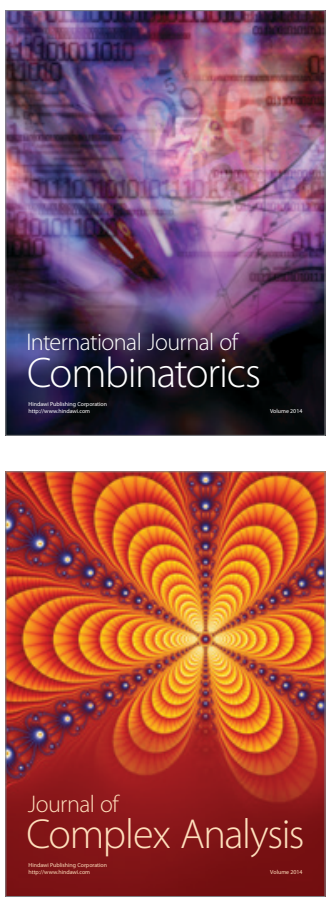

International Journal of

Mathematics and

Mathematical

Sciences
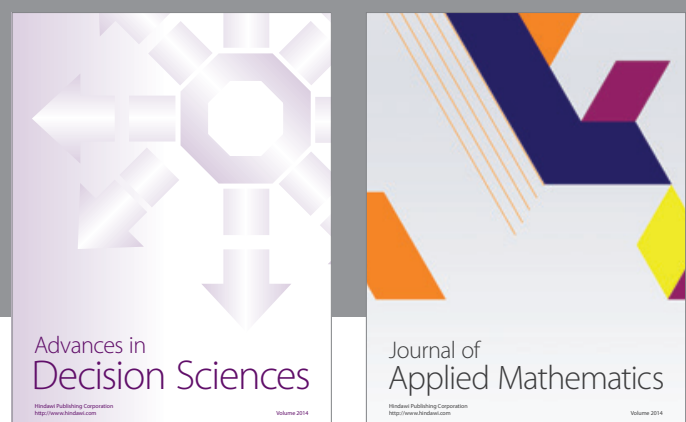

Journal of

Applied Mathematics
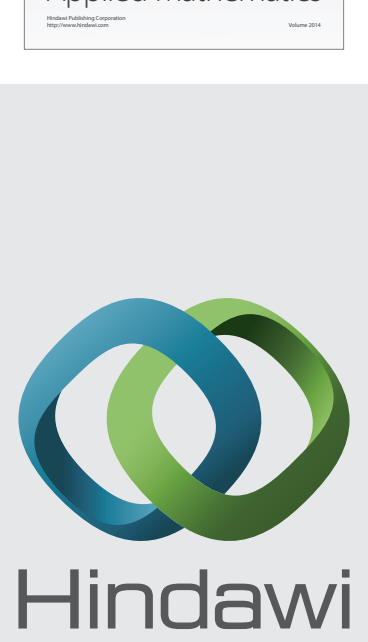

Submit your manuscripts at http://www.hindawi.com
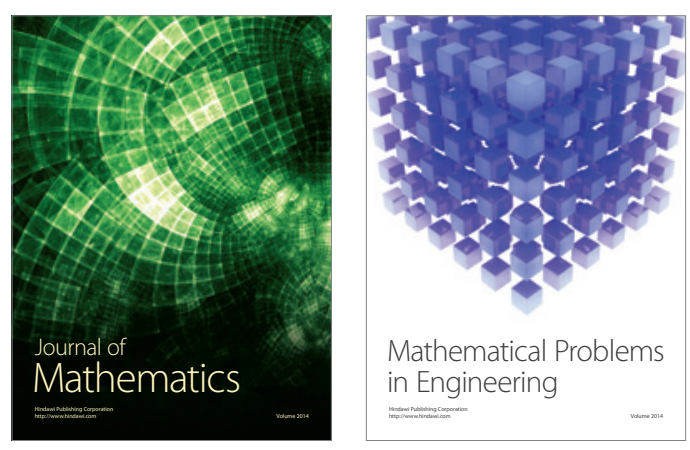

Mathematical Problems in Engineering
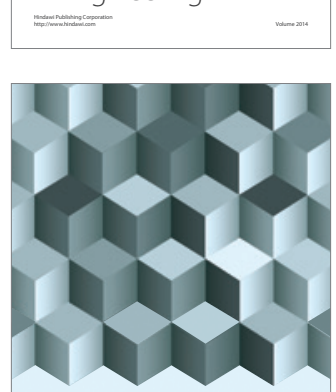

Journal of

Function Spaces
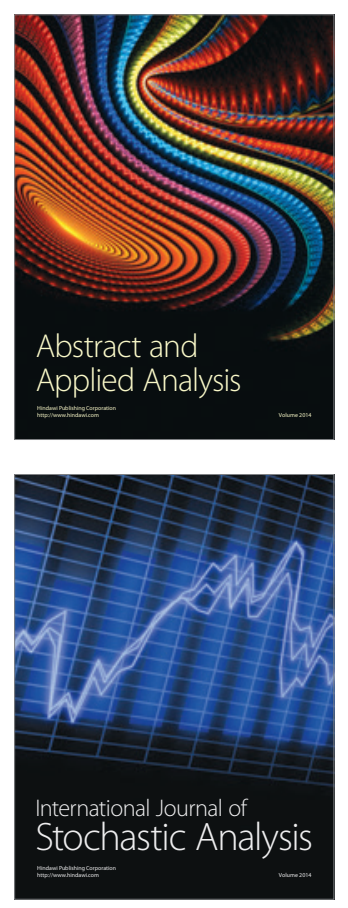

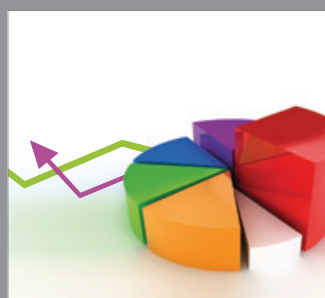

ournal of

Probability and Statistics

Promensencen
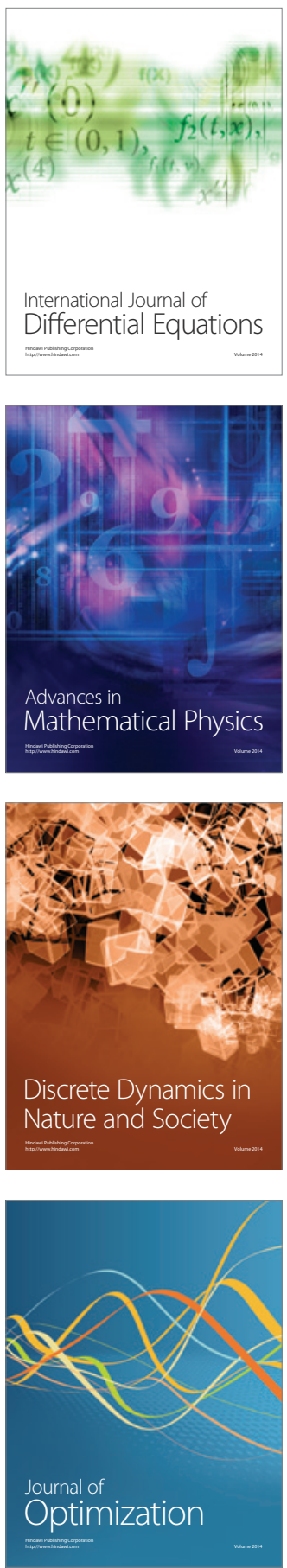